\title{
Coupling Coefficients for Coupled-Cavity Lasers
}

\author{
ROBERT J. LANG AND AMNON YARIV
}

\begin{abstract}
We derive simple, analytic formulas for the field coupling coefficients in a two-section coupled-cavity laser using a local field rate equation treatment. We show that there is a correction to the heuristic formulas based on power flow calculated by Marcuse; the correction is in agreement with numerical calculations from a coupled-mode approach.
\end{abstract}

YOUPLED-CAVITY lasers are of interest both as single-mode sources and as examples of optical feedback into a laser. In analytic treatments which treat the system as two interacting cavities, the coupling coefficients between the cavities are of paramount importance. Thus far, they have been derived in one of two ways, either heuristically, from considerations of power flow, or numerically, within the framework of a coupled-mode analysis [1], [2]. In this letter, we calculate simple analytic formulas for the intercavity coupling coefficients using local field theory [3] and compare them to heuristic and coupled-mode theories.

\section{THEORY}

In a previous work [3], we showed how to derive rate equations for traveling-wave field amplitudes from steadystate relations derived from continuity conditions. The method can be summarized briefly as follows. Given a set of $N$ field amplitudes $E_{i}$ measured at fixed points within a resonator system, one can assume $\exp (j \Omega t)$ dependence and write a set of $N \Omega$-dependent linear equations linking the field amplitudes. By treating the different amplitudes $E_{i}$ as components of an $N$-vector $\tilde{E}$, the equations can be put into matrix form as

$$
\tilde{\tilde{\Gamma}}(\Omega) \tilde{E}=\tilde{O}
$$

where the elements of the $N \times N$ matrix $\tilde{\tilde{\Gamma}}$ are the coefficients of the equations and $\tilde{O}$ is the zero vector.

Given this system, a set of rate equations for the amplitudes $E_{i}$ of a field oscillating at frequency $\Omega_{0}$, valid for fluctuations on a time scale longer than that corresponding to the longitudinal mode spacing (in single-mode lasers, this covers all frequencies of interest), is

$$
\frac{d}{d t} \tilde{E}=j \tilde{\tilde{K}} \tilde{E} \quad \text { where } \tilde{\tilde{K}} \equiv-\left.\tilde{\tilde{\Gamma}}_{\Omega}^{-1} \tilde{\tilde{\Gamma}}\right|_{\Omega=\Omega_{0}} .
$$

$\tilde{\tilde{\Gamma}}_{\Omega} \equiv \partial \tilde{\tilde{\Gamma}} / \partial \Omega$, and $\Omega_{0}$ is close to a root of the equation $\operatorname{det} \tilde{\tilde{\Gamma}}(\Omega)=0$.

Manuscript received June 5, 1986.

The authors are with the California Institute of Technology, Pasadena, CA 91125.

IEEE Log Number 8612560

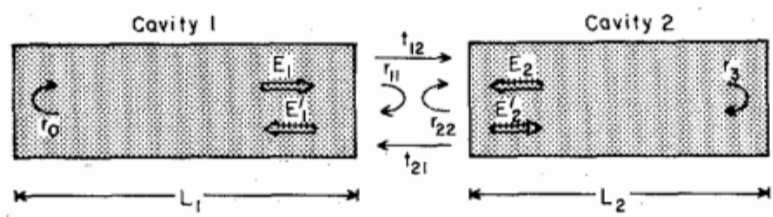

Fig. 1. Schematic of a two-section coupled-cavity laser. The gap is characterized by reflectivities $r_{11}$ and $r_{22}$ and transmissivities $t_{21}$ and $t_{22}$. The reflectivities at the ends are $r_{0}$ and $r_{3}$. The lengths are $L_{1}$ and $L_{2}$, and the (complex) indexes of refraction (incorporating gain or loss) are $\mu_{i}$ and $\mu_{2}$. All fields are measured at the gap in each laser cavity. Primed fields are the amplitudes of the waves traveling away from the gap; unprimed fields are the amplitudes of the waves traveling toward the gap.

For the two-section coupled-cavity laser illustrated in Fig. 1, we choose the field amplitudes $E_{1}$ and $E_{2}$ measured at the gap. The continuity conditions can be written as [4]

$$
\left(\begin{array}{ll}
\frac{e^{-\varphi 1}}{r_{0} r_{11}}-1 & t_{21} r_{11} \\
\frac{t_{12}}{r_{22}} & \frac{e^{-\varphi 2}}{r_{3} r_{22}}-1
\end{array}\right)\left(\begin{array}{l}
E_{1} \\
E_{2}
\end{array}\right)=\left(\begin{array}{l}
0 \\
0
\end{array}\right)
$$

where $\varphi_{1} \equiv-2 j \Omega \mu_{1} L_{1} / c, \varphi_{2} \equiv-2 j \Omega \mu_{2} L_{2} / c$, and $\mu_{i}$ and $L_{i}$ are the index of refraction and length of the $i$ th cavity, respectively. The index can be complex to accommodate gain and loss. $t_{12}, t_{21}, r_{11}$, and $r_{22}$ are the transmission and reflection coefficients of the gap. Since typical gaps are only a few wavelengths long, we will assume that the gap scattering parameters are constant over the frequencies of interest. The secular equation determining $\Omega_{0}$ is

$$
\left[\frac{e^{-\varphi 1}}{r_{0} r_{11}}-1\right]\left[\frac{e^{-\varphi_{2}}}{r_{3} r_{22}}-1\right]=\frac{t_{12} t_{21}}{r_{11} r_{12}} .
$$

Application of (2) yields the set of coupled rate equations

$$
\begin{aligned}
& \frac{d E_{1}}{d t}=j \kappa_{11} E_{1}+j \kappa_{21} E_{2} \\
& \frac{d E_{2}}{d t}=j \kappa_{22} E_{2}+j \kappa_{12} E_{1}
\end{aligned}
$$

with the self-coupling and cross-coupling coefficients given by

$$
\begin{aligned}
& \kappa_{11}=\frac{c}{2 j \mu_{1} L_{1}}\left(r_{0} r_{11} e^{\varphi_{1}}-1\right) \\
& \kappa_{22}=\frac{c}{2 j \mu_{2} L_{2}}\left(r_{3} r_{22} e^{\varphi_{2}}-1\right) \\
& \kappa_{21}=\frac{-c}{2 j \mu_{1} L_{1}} t_{21}\left(r_{0} e^{\varphi_{1}}\right)
\end{aligned}
$$




$$
\kappa_{12}=\frac{-c}{2 j \mu_{2} L_{2}} t_{12}\left(r_{3} e^{\varphi_{2}}\right) .
$$

Note that the rate equations are for field amplitudes at the mirrors; they could be normalized to some other quantity (e.g., the average photon density in each cavity), which would alter the cross-coupling coefficients. However, the only change would be to multiply one and divide the other by the same constant quantity; their product would remain fixed. We point out that these relations are fully valid for complex $\mu_{i}$ (i.e., the presence of gain and loss) and for lossy gaps.

\section{Discussion}

$E_{i}$ are the amplitudes of a field oscillating at frequency $\Omega_{0}$. If $\Omega_{0}$ is not exactly a root of the dispersion equation (4), then $E_{1}$ and $E_{2}$ will also be oscillatory. If, however, $\Omega_{0}$ is exactly a root of the dispersion equation, then the field amplitudes will be stationary and their time derivatives will be zero. In that case, we can relate the intensities at the mirrors of the light in each cavity from (5):

$$
\frac{I_{1}}{I_{2}}=\frac{\mu_{1}^{2} \mid E_{1}}{\mu_{2}^{2}\left|E_{2}\right|^{2}}=\left|\frac{\mu_{1}}{\mu_{2}}\right|^{2}\left|\frac{\kappa_{21}}{\kappa_{11}}\right|^{2}=\left|\frac{\mu_{1}}{\mu_{2}}\right|^{2}\left|\frac{\kappa_{22}}{\kappa_{12}}\right|^{2} .
$$

Obviously, the self-coupling coefficients cannot be neglected [2]; in fact, we see in $(6 a),(6 b)$ that the parenthetical quantity cannot be zero unless the right-hand side of the dispersion equation (in which it also appears) goes to zero. This right-hand side, $t_{12} t_{21} / r_{11} r_{22}$, is in some fashion a measure of the "strength" of the coupling. If the gap disappears, the coupling is perfect (and the RHS of the dispersion equation diverges); only if the transmission goes to zero does the RHS go to zero; then, as we would expect, the two cavities operate independently and the ratio of intensities either diverges or goes to zero.

Let us look at the particular case of two nearly identical cavities. We take $\mu_{1} L_{1} \approx \mu_{2} L_{2}$ and $r_{0}=r_{3}=1$. We will absorb any slight differences in length into an imaginary phase constant $\vartheta$ so that $\varphi_{1} \equiv \varphi$, but $\varphi_{2} \equiv \varphi+\vartheta$. We can define our reference planes around the gap so that $r_{11}$ $=-r_{22} \equiv r$ and $r$ is positive real. In this case, the secular equation

$$
\left(e^{-\varphi}-r\right)\left(e^{-\varphi-\vartheta}+r\right)=t_{21} t_{12}
$$

can be solved analytically to get

$$
\begin{aligned}
e^{-\varphi}= & -\frac{r\left(e^{\vartheta}-1\right)}{2} \\
& \pm\left[\frac{r^{2}\left(e^{\vartheta}-1\right)^{2}}{4}+\left(r^{2}+t_{12} t_{21}\right) e^{\vartheta}\right]^{1 / 2} .
\end{aligned}
$$

The coupling coefficients are

$$
\kappa_{21}=\frac{-c}{2 j \mu L} t_{21}\left(e^{\varphi}\right), \kappa_{12}=\frac{-c}{2 j \mu L} t_{12}\left(e^{\varphi+\vartheta}\right) .
$$

Allowing for the differences in field normalizations, these are the same as Marcuse's heuristic formulas, except for the correction factors $\exp (\varphi)$ and $\exp (\varphi+\vartheta)$. Let us examine the first correction factor. For a lossless cavity, it is a well-known result of microwave theory that the factor $t_{12} t_{21} / r_{11} r_{22}$ must be negative real. Consequently, as we have defined $r$, the product $t_{12} t_{21}$ must be positive real; thus, since $|r|^{2}+|t|^{2}=1$, the correction factor reduces to

$$
e^{\varphi}=\left\{-\frac{r\left(e^{\vartheta}-1\right)}{2} \pm\left[\frac{r^{2}\left(e^{\vartheta}-1\right)^{2}}{4}+e^{\vartheta}\right]^{1 / 2}\right\}^{-1} .
$$

(There are two choices of sign because there are two nearly degenerate longitudinal modes, one from each cavity.) For the case $\vartheta=0$, the correction simply reduces to \pm 1 , and the heuristic formulas are correct. On the other hand, if the two cavities differ by a quarter wavelength, i.e., $\vartheta=j \pi$, then we find that the correction term is

$$
e^{\varphi}=\frac{-1}{r \pm j \sqrt{1-r^{2}}}
$$

which still has magnitude 1, but a different phase from that predicted by the heuristic formulas.

However, for lossy gaps, numerical calculations show that the heuristic formulas fall short by a factor "close to one half" [2]. For a lossy gap, $|r|^{2}+|t|^{2}<1$; consequently, the correction factor is going to increase. How large will it get? As the gap losses increase, the transmission will become negligibly small compared to the reflectivity, which will approach the dielectric reflectivity of one surface of the gap. In this limit, then, we find that the correction term simply reduces to

$$
e^{\varphi}=r^{-1} \text { or }-r^{-1} e^{-\vartheta}
$$

(depending on the choice of sign), both of which have magnitude $r^{-1}$; as the transmission disappears, $r$ simply approaches the dielectric reflectivity of the first surface of the gap. For a GaAs/air interface, that reflectivity is $r=$ 0.55 , which exactly accounts for the discrepancy.

It is important to note that the correction term was real only for the cases of equal optical path length, equal gain, and equal reflectors on both lasers with a lossless gap. These are rather specialized circumstances, and are unlikely to occur in a particular device. In general, one must use the exact formulas (6a)-(6d) to be assured of the correct coupling coefficients.

For cavities of unequal length, not only will the coupling coefficients differ from the heuristic formulas, but as we can see from (6), they will, in general, be different for different longitudinal modes of the resonator system. This becomes apparent by considering the case of a "'master-slave"' combination wherein one laser is pumped much harder than the other and the coupling is weak. Take laser number 1 as the master, with laser number 2 biased well below threshold. Then roots of the dispersion equation occur for values of $\Omega_{0}$ near those where $\exp \left(-\varphi_{1}\right)$ $-r_{11} r_{0}=0$. While one coupling coefficient $\kappa_{21}$ differs only by a factor $1 / r_{11}$ from the heuristic formula 


$$
\kappa_{21}=\frac{-c}{2 j \mu_{1} L_{1}} \frac{t_{21}}{r_{11}},
$$

the other,

$$
\kappa_{12}=\frac{-c}{2 j \mu_{2} L_{2}} t_{12} r_{3} e^{-\varphi_{2}},
$$

is far smaller, and of a completely different phase from that predicted by the heuristic formulas. The longitudinal modes of the device are determined when the imaginary part of $\varphi_{1}$ is nearly an integral multiple of $2 j \pi$; however, if the two cavities are of different lengths, the imaginary part of $\varphi_{2}$ will be 1) nowhere near an integral multiple of $2 j \pi$, and 2) different for each longitudinal mode. Hence, in contrast to one's intuition, different longitudinal modes of the coupled system "see" different coupling coefficients.
The need for rate equations above and beyond the steady-state dispersion equation (2) arises in considerations of the dynamics of the device. Both the self- and cross-coupling coefficients depend upon the carrier density; knowledge of their dependence thereon is necessary for a small-signal analysis. Because of their accuracy and simplicity, the formulas in (6) are more suitable to such treatments than either heuristic or numerical formulas.

\section{REFERENCES}

[1] D. Marcuse, "Coupled mode theory of optical resonant cavities," IEEE J. Quantum Electron., vol. QE-21, pp. 1819-1826, Nov. 1985.

[2] - "Coupling coefficients of coupled laser cavities," IEEE J. Quantum Electron., vol. QE-22, pp. 223-226, Feb. 1986.

[3] R. J. Lang and A. Yariv, "Local field rate equations for coupled optical resonators,"' Phys. Rev. A, vol. 34, pp. 2038-2043, 1986.

[4] C. H. Henry and R. F. Kazarinov, "Stabilization of single-frequency operation of coupled-cavity lasers," IEEE J. Quantum Electron., vol. QE-20, pp. 733-744, July 1984. 